\title{
Targeted treatment tested as potential cancer cure
}

\section{Trial will deploy genetically targeted therapy early, rather than as last resort.}

BY ERIKA CHECK HAYDEN

A fter Van VanderMeer was diagnosed with advanced lung cancer, the results of a genetic test offered some hope. Last year, the 64-year-old lawyer learned that his cancer featured a genetic rearrangement that might render it vulnerable to a drug being tested in clinical trials. But because the experimental drug, crizotinib, was being given only to patients who had failed chemotherapy, VanderMeer had to wait for more than a year to gain access to the drug. Even though VanderMeer's tumours had by then spread to both of his lungs, crizotinib vaporized them within two weeks.

VanderMeer is now doing well and hoping to continue beating the disease: more than half of patients who take the drug, made by Pfizer of New York, seem to have a better prognosis than do those who didn't receive treatment. But what if VanderMeer had started taking it sooner?

Now oncologists, pathologists and geneticists are hoping to answer that question with a study that will test whether genetically targeted treatments, applied soon enough, can cure patients of lung cancer rather than buying them a few extra months of life.

Targeted therapies have now been approved for many cancers, and it has become routine for major cancer centres to genotype patients' tumours to determine whether they might benefit from targeted drugs, in case standard treatments fail. But the clinical trial, which will be conducted by the Alliance for Clinical Trials in Oncology, a nationwide group funded by the US National Cancer Institute in Bethesda, Maryland, will test whether using targeted treatments earlier can prevent patients with lung cancer from ever reaching that point.

In the trial, tumours will be genotyped after surgery to determine whether mutations are

\section{IDENTIFYING TARGETS}

Genotyping of lung tumours from more than 500 patients revealed genetic changes that could be targeted by drugs. Some patients had more than one mutation. KRAS
$\mathbf{2 4 \%}$

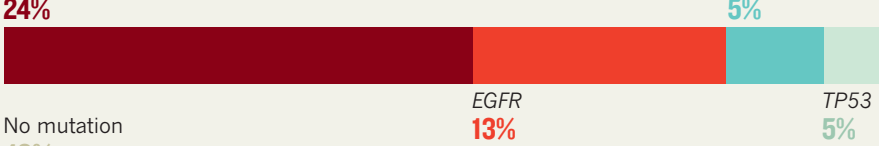
No mutation $13 \%$

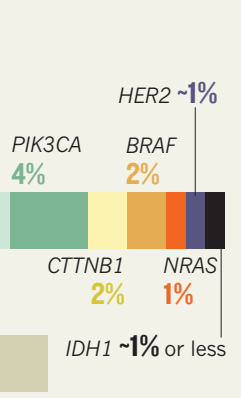

present in a gene encoding epidermal growth factor receptor (EGFR). Mutations in this gene are targeted by many molecular therapies, including erlotinib and gefitinib, which are approved for the treatment of advanced lung cancer. Some of the patients who have EGFR mutations will begin taking erlotinib after surgery, instead of waiting to see whether their cancer recurs.

Although similar approaches have been tested in smaller trials, yielding mixed results, organizers say that a larger, better-defined study is needed to provide a clear answer.

"We have never tested these drugs in the right population," says oncologist Ramaswamy Govindan of Washington University in St Louis, leader of the trial. "We have never tested a group of patients who have mutations in EGFR and then asked the question, 'could these patients be cured by gefitinib or erlotinib?'”

He hopes to expand the analysis to include crizotinib, which targets a different genetic rearrangement and was approved by the US Food and Drug Administration in August. Other targeted therapies are in the pipeline. In a 9 November paper, for instance, a consortium of researchers from Massachusetts General Hospital and Harvard Medical
School, both in Boston, and Yale University in New Haven, Connecticut, describe the results of a study that tested more than 500 patients with non-small cell lung cancer (L. V. Sequist et al. Ann. Oncol. http://dx.doi.org/10.1093/ annonc/mdr489; 2011). The authors examined mutations in several genes relevant to therapies that have been approved or are in development (see 'Identifying targets'). Of the 353 patients with the most advanced lung cancers, $22 \%$ were matched to clinical trials appropriate for their cancer type.

The Alliance trial will be logistically difficult. Only $10-20 \%$ of patients with non-small cell lung cancer have mutations in the EGFR gene; only $20 \%$ of patients are diagnosed early enough to benefit from surgery; and only a fraction of patients with the appropriate mutations will actually gain any advantage from targeted treatments. To reach their target of 400 participants, Govindan and his colleagues may need to screen as many as 1,500 people.

VanderMeer, for one, hopes that the efforts pay off - and spare other patients from what he calls the "blunderbuss" of chemotherapy.

"I'd hate for anyone to have to go through the blunderbuss before they get to the stiletto," he says. ONLINE

EXPLAINER
The science
behind
Australia's war
on tobacco
advertising
go.nature.com/
zjrfci

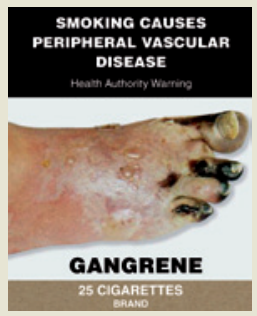

\section{MORE NEWS}

- Sickle-cell mystery solved go.nature. com/dxx6lh

- Ancient adaptations to parasites drove human genetic variation go.nature.com/d4es4b - Proof found for unifying quantum principle go.nature.com/dt8syh

\section{FROM THE BLOG}

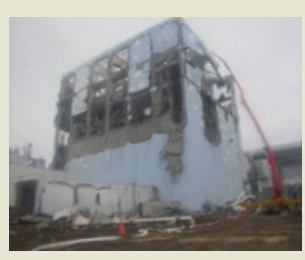

Japan

funds

Fukushima

clean-up projects

go.nature. com/wdfscp 